\title{
Ruta Bioceánica: un enfoque basado en indicadores económicos de 2016 a 2019
}

\author{
Rota Bioceânica: uma abordagem baseada em indicadores econômicos \\ de 2016 a 2019
}

Bioceanic Route: an approach based on economic indicators from 2016 to 2019

\author{
Felipe Figueiredo Fernandes Brites ${ }^{1}$ \\ Michel Constantino ${ }^{1}$ \\ Arlinda Cantero Dorsa ${ }^{1}$
}

Recibido el 7 de jul. de 2021; revisado e aprobado el 28 de sept. de 2021; aceptado el 13 de oct. de 2021 DOI: http://dx.doi.org/10.20435/inter.v22i4.3449

Resumen: La Ruta de la Integración Latinoamericana discurre por el Corredor Bioceánico, también Ilamado ruta vial, con la intención de conectar el Océano Atlántico al Océano Pacífico, pasando por países como Brasil, Paraguay, Argentina y Chile. En este contexto, el presente artículo tuvo como objetivo analizar los datos económicos del tramo Brasil-Paraguay, centrándose en la ciudad de Porto Murtinho. Cabe destacar que este trabajo está relacionado con el proyecto de investigación Corredor Vial Bioceánico y Red Universitaria de la Ruta de Integración Latinoamericana (UniRila): El análisis verificó los flujos de importaciones y exportaciones en un escenario macroeconómico que se tradujo en perspectivas del corredor, presentando retos a seguir, tales como: tasas impositivas entre países, reducción del proteccionismo, corrupción, integración de universidades e intercambio. Para Porto Murtinho y Carmelo Peralta, la expectativa es que con el crecimiento del flujo logístico y las exportaciones, el ingreso per cápita se incremente y apalanque las mejoras sociales en los municipios.

Palabras clave: Corredor Bioceánico; Ruta de la Integración Latinoamericana; Porto Murtinho; Brasil-Paraguay; UniRila.

Resumo: A Rota de Integração Latino Americana, perpassa o Corredor Bioceânico, também chamado de rota rodoviária com a intenção de conectar o Oceano Atlântico ao Oceano Pacífico passando por países como o Brasil, Paraguai, Argentina e Chile. Neste contexto, o presente artigo teve como objetivo analisar os dados econômicos do trecho Brasil-Paraguai com enfoque na cidade de Porto Murtinho. Enfatiza-se que este trabalho se relaciona ao projeto de pesquisa Corredor Rodoviário Bioceânico e Rede de universidades da Rota de Integração Latino Americana (UniRila): A análise verificou os fluxos de importações e exportações num cenário macroeconômico que traduziu em perspectivas do corredor, apresentando desafios a frente como: taxas de tributação entre países, redução do protecionismo, corrupção, integração das universidades e câmbio. Para Porto Murtinho e Carmelo Peralta, a expectativa é que com o crescimento do fluxo logístico e as exportações, a renda per capita aumente e alavanque melhorias sociais nos municípios.

Palavras-chave: Corredor Bioceânico; Rota de Integração Latino Americana; Porto Murtinho; Brasil-Paraguai; UniRila.

Abstract: The Latin American Integration Route runs through the Bioceanic Corridor, also called road route, with the intention of connecting the Atlantic Ocean to the Pacific Ocean, passing through countries such as Brazil, Paraguay, Argentina and Chile. In this context, this article aimed to analyze the economic data of the Brazil-Paraguay stretch, focusing on the city of Porto Murtinho. It is emphasized that this work is related to the research project Bioceanic Road Corridor and University Network of the Latin American Integration Route (UniRila): The analysis verified the flows of imports and exports in a macroeconomic scenario that translated into perspectives of the corridor, presenting challenges forward as: tax rates between countries, reduction of protectionism, corruption, integration of universities and exchange. For Porto Murtinho and Carmelo Peralta, the expectation is that with the growth of the logistical flow and exports, the per capita income will increase and leverage social improvements in the municipalities.

Keywords: Bioceanic Corridor; Latin American Integration Route; Porto Murtinho; Brazil-Paraguay; UniRila.

\footnotetext{
${ }^{1}$ Universidade Católica Dom Bosco, Campo Grande, Mato Grosso do Sul, Brasil.
} 


\section{INTRODUCCIÓN}

El Corredor Bioceánico es una ruta vial con la intención de conectar el Océano Atlántico con el Océano Pacífico que pasa por países como Brasil, Paraguay, Argentina y Chile. Dicha ruta facilitaría al acceso de Brasil a los mercados del este y de Chile y de Argentina a Europa Occidental, además de permitir una salida para la costa a Paraguay.

La Red Universitaria de la Ruta de Integración Latinoamericana (UniRila) se trata de una red de universidades con el propósito de fortificar la integración del Corredor Vial Bioceánico, también forma parte del objetivo de esta unión presentar la investigación relacionada con la Ruta de Integración Latino Americana para decidir las acciones futuras en las diversas áreas para su implementación en los varios países por donde el corredor pasará.

Con el objetivo de mapear los impactos de la Ruta relacionada al Corredor Bioceánico, en el tramo de Brasil-Paraguay, se busca, por tanto, analizar datos cuantitativos relativos a la economía de las localidades del segmento en la cuestión. Para el análisis de estos datos, se utilizó la Escuela Austríaca de Economía como base teórica y para los fines de análisis y solución de adversidades encontradas en la construcción del corredor.

La temática elegida procura, por lo tanto, presentar los datos relativos a los impactos causados por la ruta en el recurrido analizado, además de promover la ampliación del conocimiento referente al tema de las comunidades académicas de grado y posgrado. A fin de lograr el objetivo propuesto, el presente artículo se organiza en esta introducción, en la revisión de la literatura de la Ruta de la Integración Latino Americana y el funcionamiento Bioceânico, del UniRila y de algunos de los mecanismos de la macroeconomía necesarios para el análisis de los datos y de las adversidades encontradas.

\section{CONSIDERACIONES SOBRE EL CORREDOR BIOCEÁNICO}

El Corredor Bioceánico es una ruta vial con la intención de conectar el Océano Atlántico con el Océano Pacífico, que pasa por los países como Brasil, Paraguay, Argentina y Chile. Tal ruta facilitará al acceso de Brasil a los mercados del este y de Chile y de Argentina a Europa Occidental, además de permitir una salida para la costa a Paraguay.

Entre los objetivos de la Ruta están la reducción del tiempo de tránsito, coste de servicio del transporte, almacenaje e inventario, así como la promoción de un movimiento de carga más eficiente, más segura y previsible; estímulo a la formación de asociaciones, uso de más de un modal y el desarrollo de proyectos. integración productiva y la agregación de valor en los países de origen y de destino, bien como en los países del tránsito.

El Corredor, relacionado a la Ruta de Integración Latino Americana (RILA) posee una extensión de 2.396 kilómetros con planificaciones determinadas en cada país para donde pasa: I) en Brasil, para proveer al agro negocio una salida al Pacífico, permitiendo la destinación de la producción cuanto a la importación directa de insumos a precios más competitivos; II) en Paraguay, mejorar la infraestructura e integrar la región del Chaco al resto del país; III) en Argentina, fortificar la implementación del Plan Belgrano, que prevé inversiones en la infraestructura de la orden de US\$ 15 mil millones, en la medida donde cruza las provincias de Salta y de Jujuy; IV) en Chile, desarrollar el comercio con los países de la región, siendo consolidado el país como plataforma logística importante ${ }^{2}$.

\footnotetext{
${ }^{2}$ Ver más en https://ecoa.org.br/rota-bioceanica-o-que-e-e-seus-impactos-diretos-e-indiretos.
} 


\subsection{Las acciones de la Red de Universidad de la Ruta de Integración Latinoamericana (UniRila)}

El principio de su desarrollo fue a mediados de 2016, no obstante, su fundación vino a materializarse en el Seminario UniRila en octubre de 2017. La UniRila se trata de una red de la universidad con el propósito de fortificar la integración del Corredor Vial Bioceánico. También forma parte del objetivo de esta unión presentar investigaciones relacionadas con la Ruta de Integración Latino Americana que puedan colaborar decisivamente con las acciones futuras en diversas áreas, para suu implementación en los países por donde pasará el Corredor.

La Red de Universidades de la Ruta de Integración Latino Americana se compone por la Universidad Católica Dom Bosco (UCDB), la Universidad del Estado de Mato Grosso de Sul (UEMS), la Universidad Federal de Mato Grosso de Sul (UFMS), la Universidad Federal de Grande Dourados (UFGD), la Universidad de Anhanguera/Uniderp, el Instituto Federal de Mato Grosso de Sul (IFMS), todas estas de Brasil. Universidad Nacional de Jujuy (Argentina), Universidad Nacional de Salta y Universidad Católica de Salta (Argentina), Universidad de Antofagasta (Chile), Universidad Católica del Norte de Chile y la Universidad Nacional de Asunción (Paraguay).

Con la formación de esta red universitaria, desde 2017, varias acciones ya han sido realizadas, entre ellas: reuniones en persona en Salta, Asunción y Brasil, reuniendo a representantes del gobierno, de entidades privadas, de asociaciones, así como de los representantes de la red de universidades.

Con respecto a la UniRila, además de encuentros efectivos a partir de lives llevados a cabo entre los cuatro países, fueron publicados artículos científicos disponibles en los respectivos enlaces: I) I Expediente "Desafios da Integração en la Ruta Bioceánica (Brasil, Paraguay, Argentina y Chile)", disponibles en la Revista Interações ${ }^{3}$; la publicación II) Instituto de Pesquisa Econômica Aplicada/ Universidade Estadual de Mato Grosso do Sul (IPEA/UEMS) intitulada: El Corredor Vial Bioceánico entre Porto Murtinho en Mato Grosso del sur y los puertos del norte de Chile 4 . Para 2021, está en marcha el II Expediente "Desafíos da Integração na Rota Bioceânica (Brasil, Paraguay, Argentina y Chile)" con la publicación prevista en diciembre, de los artículos publicados en español o inglés.

Hay planes para un acuerdo multilateral entre las Universidades para la cooperación académica de profesores investigadores, además de las posibilidades infinitas que tal unión puede y proporciona para todos los países de la ruta y para los académicos, los profesores y los investigadores de todas las universidades implicadas.

\section{PROCEDIMIENTO METODOLÓGICO}

La investigación realizada tuvo enfoque cuantitativo y los procedimientos metodológicos contemplaron necesariamente una investigación detallada de los datos socioeconómicos de los países y de las ciudades incluidas por la ruta, en la recogida de datos, organización y elaboración del considerado del mapeo propuesto. El foco de este trabajo, en efecto, ha sido señalar las posibilidades de crecimiento de las ciudades y de los países implicados en el tramo delimitado, incluso, las adversidades al desarrollo económico.

\footnotetext{
${ }^{3}$ Ver más en https://interacoesucdb.emnuvens.com.br/interacoes/issue/view/124.

${ }^{4}$ Ver más en https://www.ipea.gov.br/portal/images/stories/PDFs/livros/livros/210504_livro_corredor_bioceanico_ipea_ uems_2020.pdf.
} 
Para tal, los tipos de productos exportados e importados para los países en cuestión y la relación entre estas exportaciones e importaciones con los demás países que pertenecientes a la Ruta, dispuestos por el The Observatory of Economic Complexity (OEC) en el año de 2019; datos económicos de la ciudad de Porto Murtinho, dispuestos por la Secretaria de Estado de Meio Ambiente, Desenvolvimento Econômico, Produção e Agricultura Familiar de Mato Grosso do Sul (SEMAGRO/MS), tales como el Produto Interno Bruto (PIB) y el PIB per cápita y su desarrollo a lo largo de los años en detrimento a la Ruta de Bioceánica que compara el período antes de la ruta (2010-2015) con durante su pre-construcción (2016-2018).

\subsection{Teoría económica}

Para el análisis de la teoría económica ha sido la Escuela Austríaca de Economía. Según la teoría, los valores de productos y los servicios son determinados por una evaluación subjetiva, por otra parte su blanco está a interdisciplinar y su metodología es subjetiva, individualista y aplica la lógica deductiva para llegar a sus conclusiones. Amplía esta discusión, Huerta de Soto al afirmar que:

Estos principios serían, esencialmente, el desarrollo de una ciencia económica basada en el humano considerado como agente creativo y protagonista de todos los procesos y acontecimientos sociales (Subjetivismo), bien como la elaboración, en base al Subjetivismo, y por primera vez en la historia del pensamiento económico, de toda una teoría formal sobre el aparecimiento y la evolución de todas las instituciones sociales (económicas, legales y lingüísticas) entendidas como esquemas pautados del comportamiento. (HUERTA DE SOTO, 2010, p. 59).

La tríada de la Escuela Austríaca de Economía es formada por la acción, el tiempo y el conocimiento, y según lorio (2011, p. 17), "Esos tres elementos forman su núcleo fundamental y se trasmite por medio de sus elementos de propagación para los diversos campos del conocimiento humano".

El método de análisis recae sobre las consecuencias de las acciones realizadas por las personas, gobiernos y empresas para entender las motivaciones y los incentivos para la toma de decisión y las consecuencias de tales actos, sean ellos positivos o negativos.

En el contexto del Corredor Bioceánico, esta teoría viene proporcionar la reducción de costes en el transporte y la mayores sencillez y seguridad en la exportación y la importación de productos, por lo tanto, es tener de los países implicados para proporcionar tal desarrollo y a no remar contra los impuestos más grandes imponentes de la marea o la fabricación le difícil de la importación para aumentar prescripciones de las cajas públicas o para favorecer a los productores nacionales.

Por diversas veces se usa por parte de los gobiernos el argumento de la "Balanza Comercial Favorable" a fin de justificar la producción nacional y prevenir mayores costos con la importación. Sin embargo, esta terminología se originó en los errores del mercantilismo que se perpetúan hasta nuestros días.

Al imponer tasas para la importación, el Estado proporciona el incremento del precio de los productos importados para la población. Para los productos electrónicos, tales como smartwatches, móviles o el hardware, no hay tanto impacto, ya que no son productos necesarios para la supervivencia. Sin embargo, cuando los impuestos se ponen en productos agrícolas, remedios y/o maquinaria, la subida de precio de los alimentos, especialmente para los de poca renta, se convierte en un problema para la supervivencia de la población. 
Actualmente hay países y ciudades profundamente dependientes del comercio de exportaciones y, con el advenimiento de la Ruta y de las actuales relaciones internacionales, nuevas ciudades pueden crecer y desarrollarse, gracias a las exportaciones. Cada vez más se ha combatido el proteccionismo y, para una economía sana, esto es bueno.

El propósito de los teóricos del libre comercio no fue demostrar que los aranceles son impracticables o dañinos, sino que tienen consecuencias imprevistas y no logran, ni pueden lograr, lo que sus defensores esperan de ellos. También señalaron que los aranceles proteccionistas, así como todas las demás restricciones de producción, reducen la productividad de la fuerza laboral humana, lo que es aún más significativo. El resultado es siempre lo mismo: una determinada inversión de capital y trabajo rinde menos con la restricción que sin ella, es decir, desde el principio, se invierte menos capital y trabajo en la producción. (MISES, 2015, p. 22).

Se puede decir, por tanto, que la RILA está en el camino cierto para el desarrollo de todos los países implicados en el proyecto. Sin embargo, se debe cuidar para que la avaricia no corrompa la evolución de la economía en América Latina.

\section{ANÁLISIS DE DATOS}

Para este análisis, en primer plano, se buscaron los productos y países con los que Brasil y Paraguay tienen grandes relaciones comerciales, disponibles en el sitio web oec.world. En segundo plano, un análisis de los datos socioeconómicos dispuestos por la SEMAGRO/MS sobre Porto Murtinho y la influencia de la ruta sobre la ciudad, además de los futuros impactos positivos y negativos en el lugar.

\subsection{Importaciones y exportaciones de Brasil y de Paraguay}

Figura 1 - Relación de las exportaciones de Brasil: productos/países - 2019

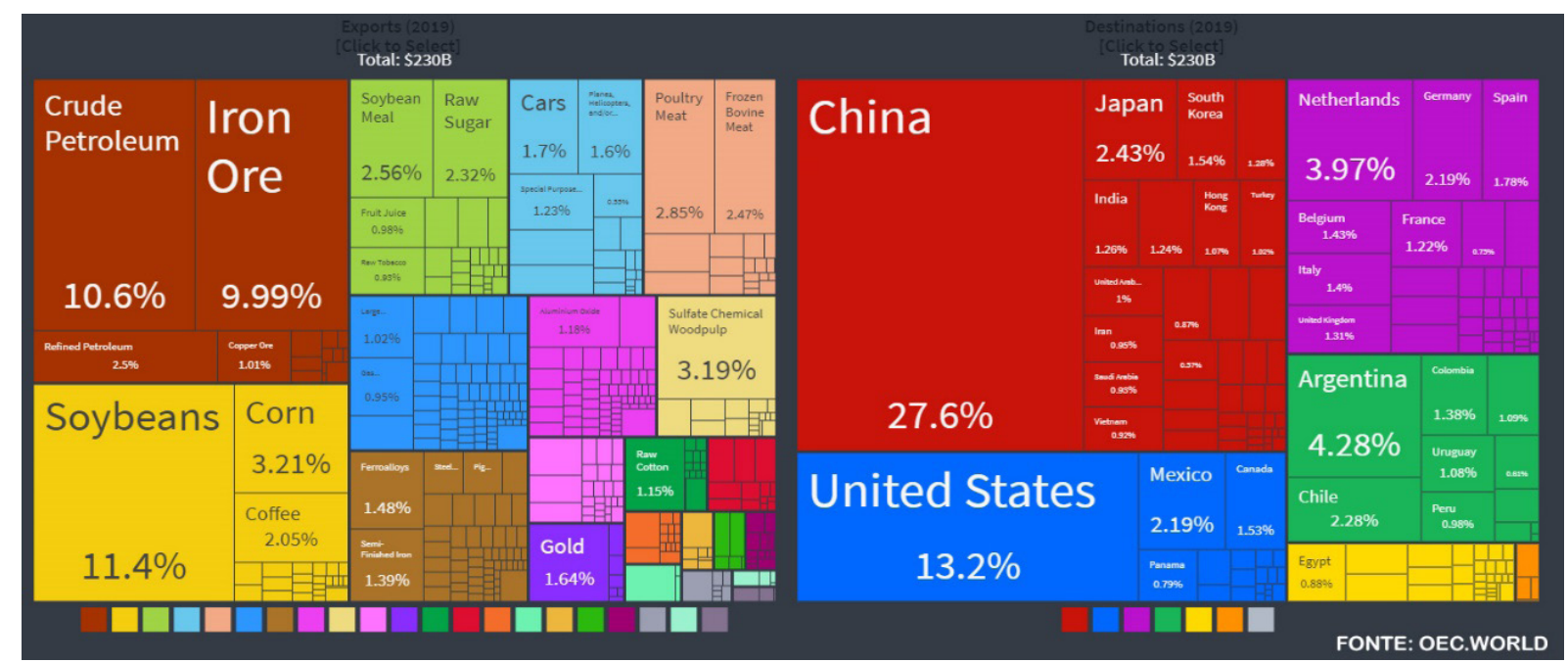

Fuente: The Observatory of Economic Complexity [OEC] (2019).

Al analizar el gráfico, se puede notar que China e el socio comercial más grande de Brasil y directamente interesado en la construcción del corredor, puesto que tendrá un menor coste en el transporte de mercancías. En la América del Sur, los socios más grandes de Brasil son 
Argentina y Chile, también directamente conectados a la ruta. Paraguay corresponde al 1.09\% de las exportaciones de Brasil que incorporan un valor de 2.5 mil millones de dólares. De las exportaciones para China, entre los principales productos están la soja (32,2\%), el petroleo bruto $(24,4 \%)$ y el minerio de hierro $(21,4 \%)$, en suma, principalmente materia prima.

Figura 2 - Países de quiénes el Brasil importa - 2019

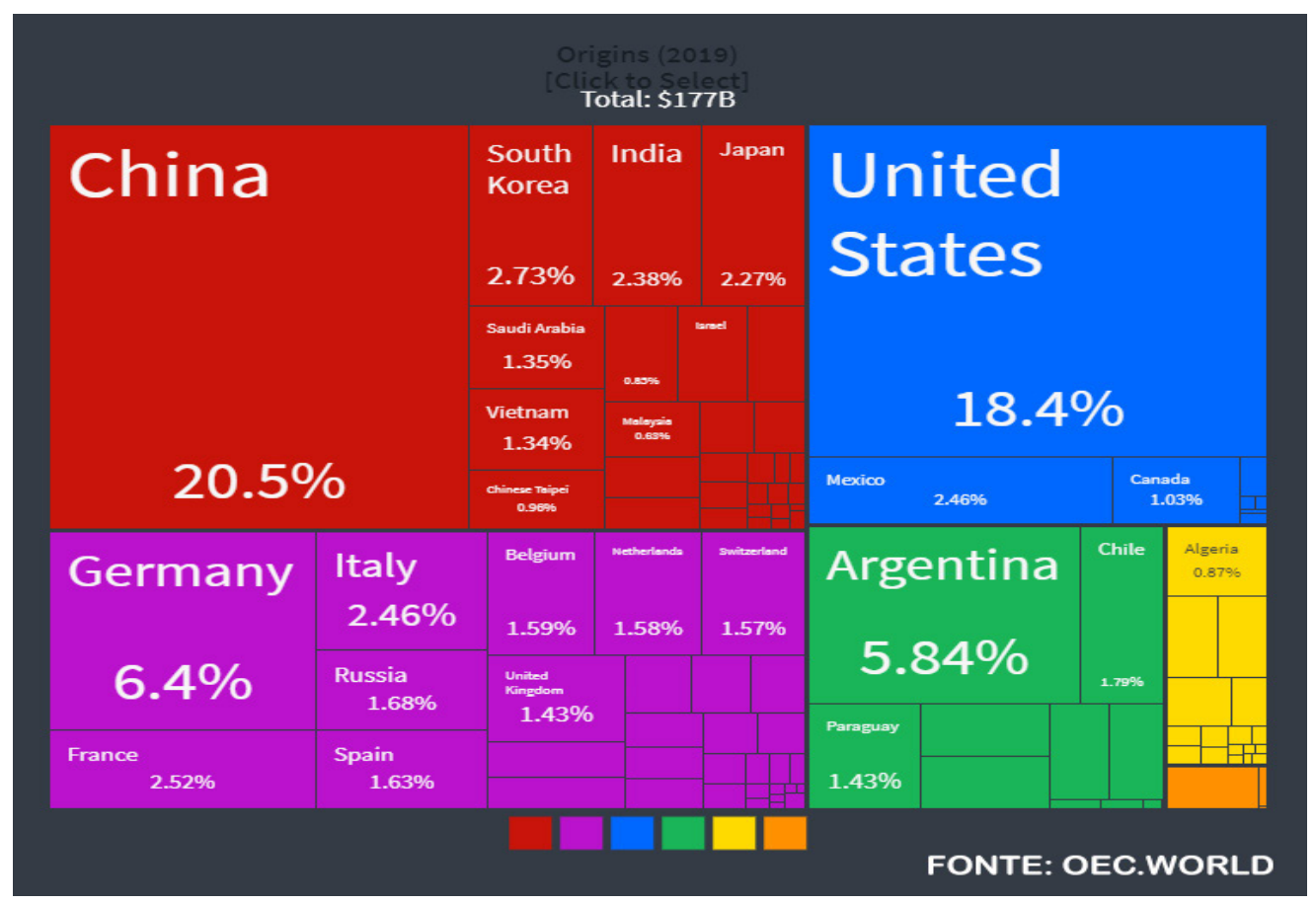

Fuente: OEC (2019).

Al analizar las regiones de países de los cuales Brasil importa, se nota un dominio de Asia, seguida de Europa y después Norteamérica. A pesar del orden establecido en el gráfico, la relevancia de América del Norte es mucho mayor que la de Europa, debido al número de países en sus respectivas regiones. Estados Unidos es el segundo mayor exportador para Brasil, con el peso de toda América del Norte depositado solo en él. Ya en Asia, China es la que más que exporta a Brasil, seguida de Corea del Sur, Japón e India. En el continente europeo, Alemania es el principal exportador para Brasil, seguida de Francia y de Italia. Entre los vecinos de Sudamérica, el más relevante es Argentina.

Con relación a los productos importados, según datos del The Observatory of Economic Complexity de 2019, de China la predominancia es de máquinas como teléfonos (3,45\%), navíos con propósitos especiales (7,96\%) y demás piezas de maquinaria que llegan en el país dirigidos a las respectivas montadoras dentro de Brasil. De Estados Unidos, Brasil importa especialmente el petróleo fino $(25,1 \%)$, además de otros productos minerales, siendo la clase que más destaca. La segunda clase que más destaca es la de productos químicos como pesticidas (2,74\%). De Alemania, hay un destaque mayor para las máquinas y las partes de los vehículos (7,43\%), además de productos químicos relacionados a la salud, como las medicinas (5,77\%). De Argentina, el destaque está para los camiones $(24,8 \%$ ) y carros (12,8\%), que son montados dentro del país vecino, además del trigo (11,3\%), que también aparece de forma relevante. 
Figura 3 - Relación de las exportaciones de Paraguay: Productos/países - 2019

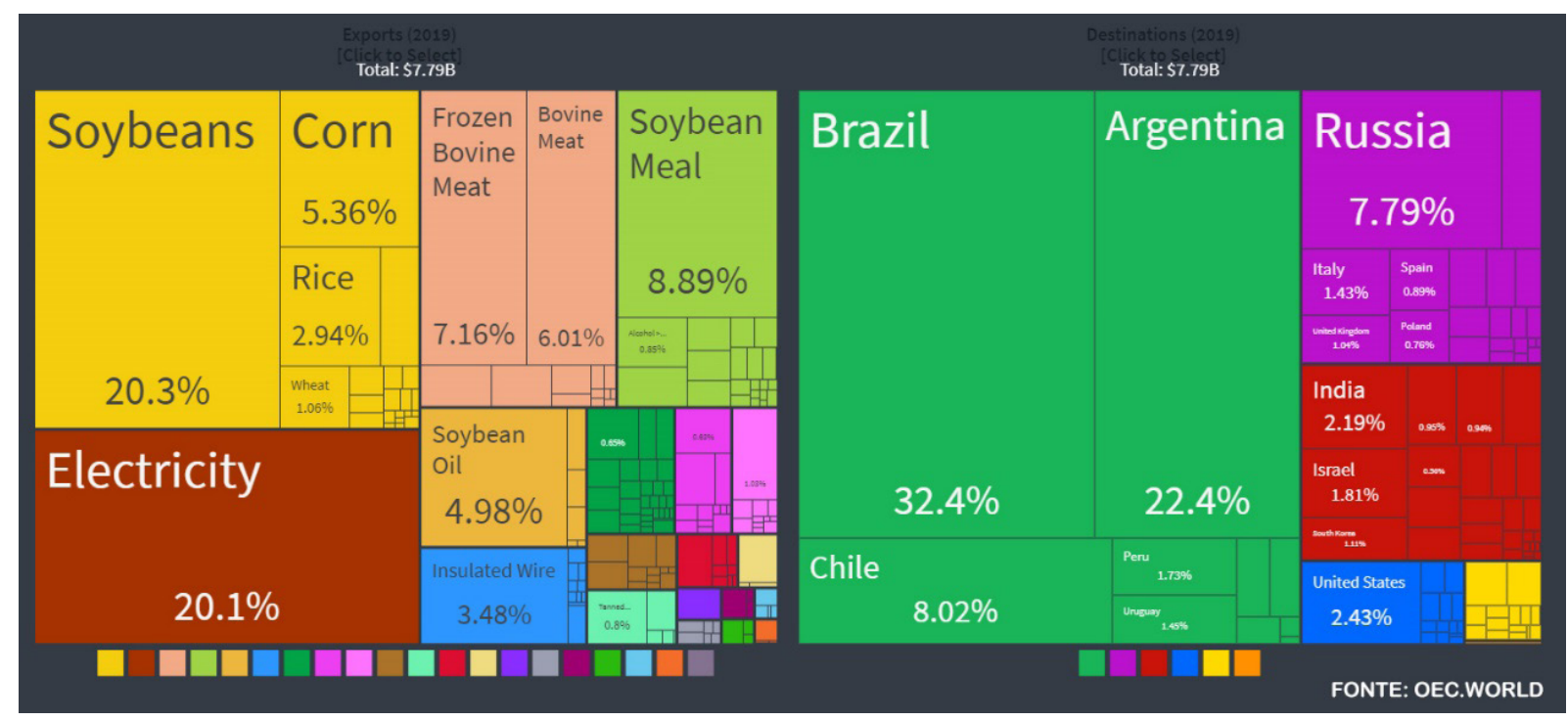

Fuente: OEC (2019).

Analizando el gráfico, se nota una influencia de América del Sur, mayor que las grandes potencias mundiales de hoy, como China y Estados Unidos, como destinos de las exportaciones desde Paraguay. Una de las razones posibles es la falta de una salida para la costa, lo que acaba por dificultar la exportación a los países que no están en el continente. Con el advenimiento de la ruta, el gráfico 2018 puede tener un gran cambio en las destinaciones de las exportaciones y, con el desarrollo de la región, incluso de los productos a ser exportados.

De la relación entre producto exportado y el destino, hay una gran exportación de soja para Argentina $(61,9 \%)$ y de electricidad (21,5\%). Para Chile hay un dominio de la carne de res $(56,9 \%)$ y de la harina de soja (26,9\%), mientras que para Rusia las exportaciones se centran en la carne de res congelada $(48,2 \%)$ y en la soja $(40,6 \%)$. Brasil ya posee una mayor variedad en lo que recibe de Paraguay (valores por encima del $5 \%$ ). Destaque principal para la electricidad $(47,2 \%)$; en productos agrículas destaca el maíz (7,23\%) y el arroz (6,02\%); y entre las máquinas, el cable aislado (9,06\%). 
Figura 4 - Países de los que Paraguay importa - 2019

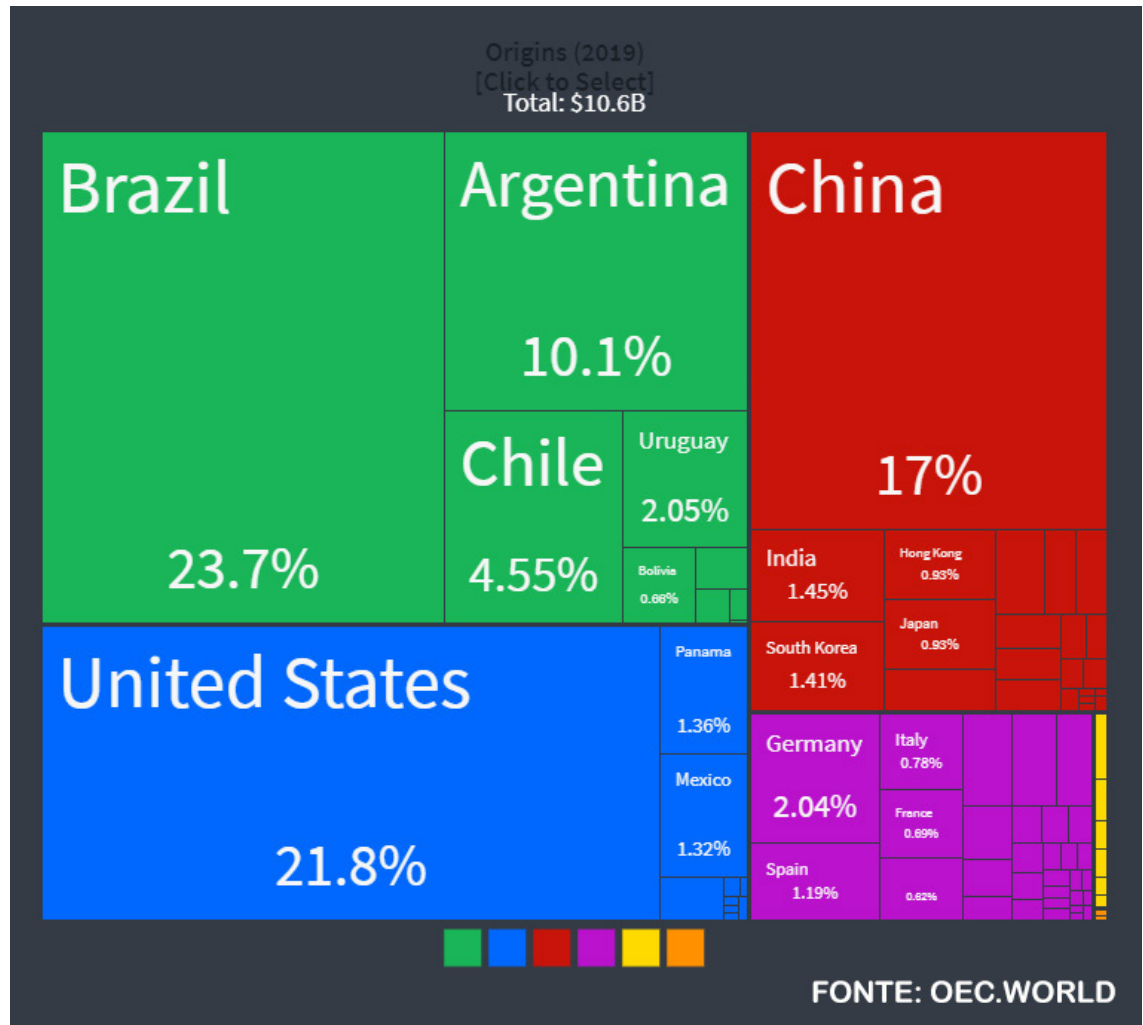

Fuente: OEC (2019).

Así como evidenciado en el gráfico de las exportaciones de Paraguay (Cuadro 3), Suramérica demuestra la importancia para el país, siendo estupendos socios comerciales, una vez que hay tanto una relación de gran importación como de la exportación, siendo Brasil el principal país exportador.

El principal producto importado por Paraguay son los equipos de comunicación (14,3\%). Su importación proviene principalmente de Estados Unidos (77,3\%), seguido de China (13,2\%), con una pequeña participación de Vietnam (2,65\%) y Hong Kong (2,15\%). 
Figura 5 - Importaciones de Paraguay provenientes de Brasil - 2019

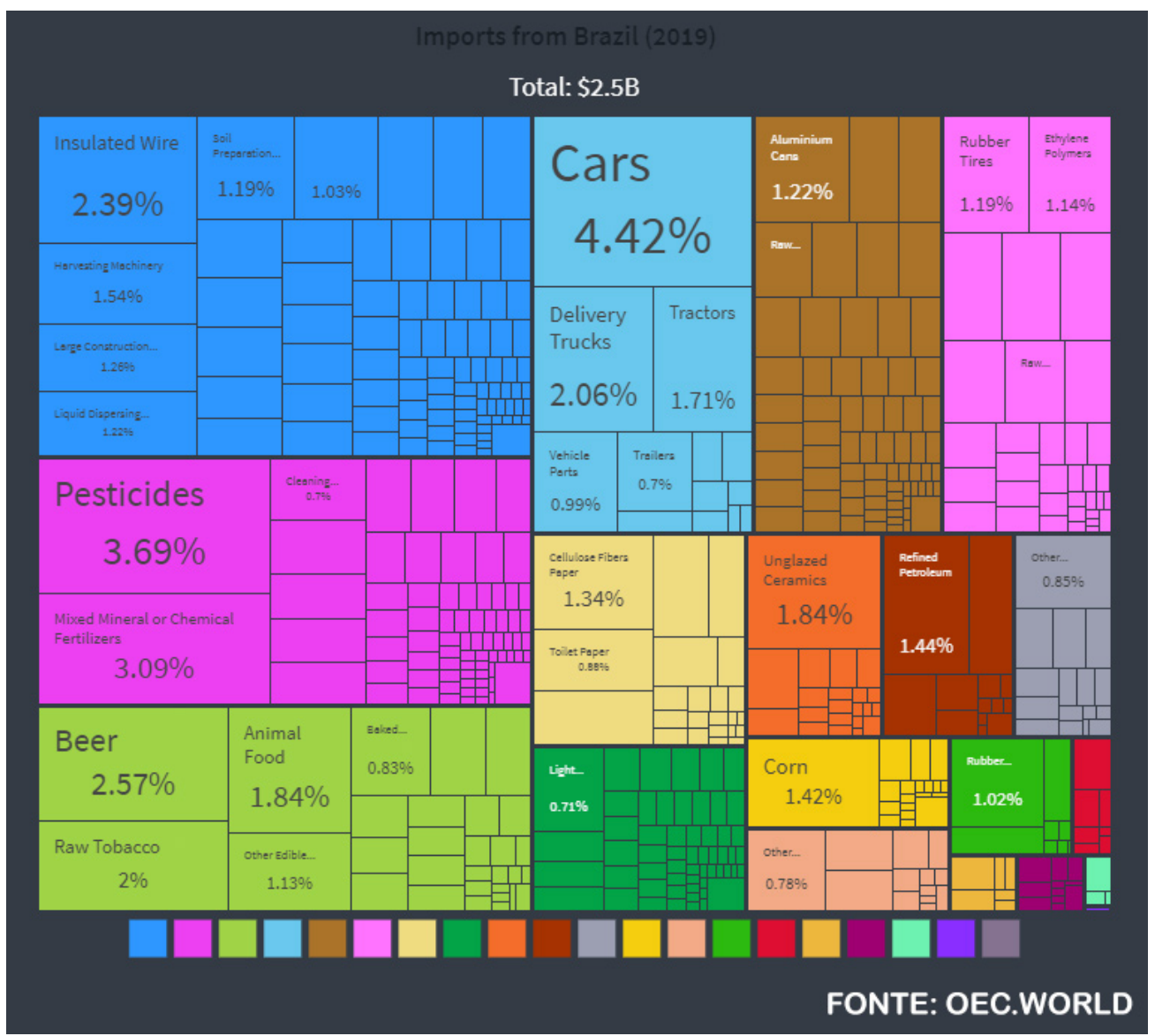

Fuente: OEC (2019).

Al analizar el gráfico, que también puede entender como las exportaciones de Brasil para Paraguay, uno nota una gran variedad de productos con destaque para el grupo de maquinarias, alimentos y de productos químicos. Debido a esta gran variedad, Paraguay es un socio comercial excelente para Brasil puesto que promueve los diversos sectores de la economía dentro del país.

A diferencia de la relación con los países más desarrollados, Brasil exporta a Paraguay productos aún del sector primario, pero hay nuevos tipos como maquinaria agrícola, automóviles, tractores y fertilizantes. La agricultura y la ganadería no se excluyen, pero su presencia es mucho más pequeña en lo referente a los países desarrollados, apareciendo aquí con el maíz, el tabaco, la cebada y la carne animal.

A partir de los datos presentados hasta el momento y al analizar la condición geográfica de los países respectivos, el Corredor Bioceánico es una ventaja mucho más grande para Paraguay que para Brasil. Permitiendo una salida más barata al mar y pudiendo aumentar sus relaciones comerciales con Asia, Paraguay tiene mucho que ganar para el país como un todo. Importante recordar el tamaño de Paraguay que, por no ser muy grande, depende mucho de productos importados para proveer sus necesidades tecnológicas y, aún contando con una posición geográfica excelente para las exportaciones a América latina, sus vecinos competentes ofrecen mayor infraestructura a abrigar las grandes plantas. 
Con el desarrollo en el potencial proporcionado por la ruta en el país y la sencillez del transporte permitida por esta, le cabe al poder pública garantizar la simplicidad a los empresarios para generar abundancia al país y, principalmente, a la gente.

En cambio Brasil, por el hecho de tener la costa extensa y el tamaño continental, no tendrá proporcionalmente como el de Paraguay, sin embargo, para las regiones locales como Porto Murtinho, habrá grandes progresos e impactos enormes para las regiones del interior relacionado directa e indirectamente con la ruta.

\subsection{Datos socioeconómicos de Porto Murtinho}

Figura 6 - PIB Porto Murtinho $2010-2018$

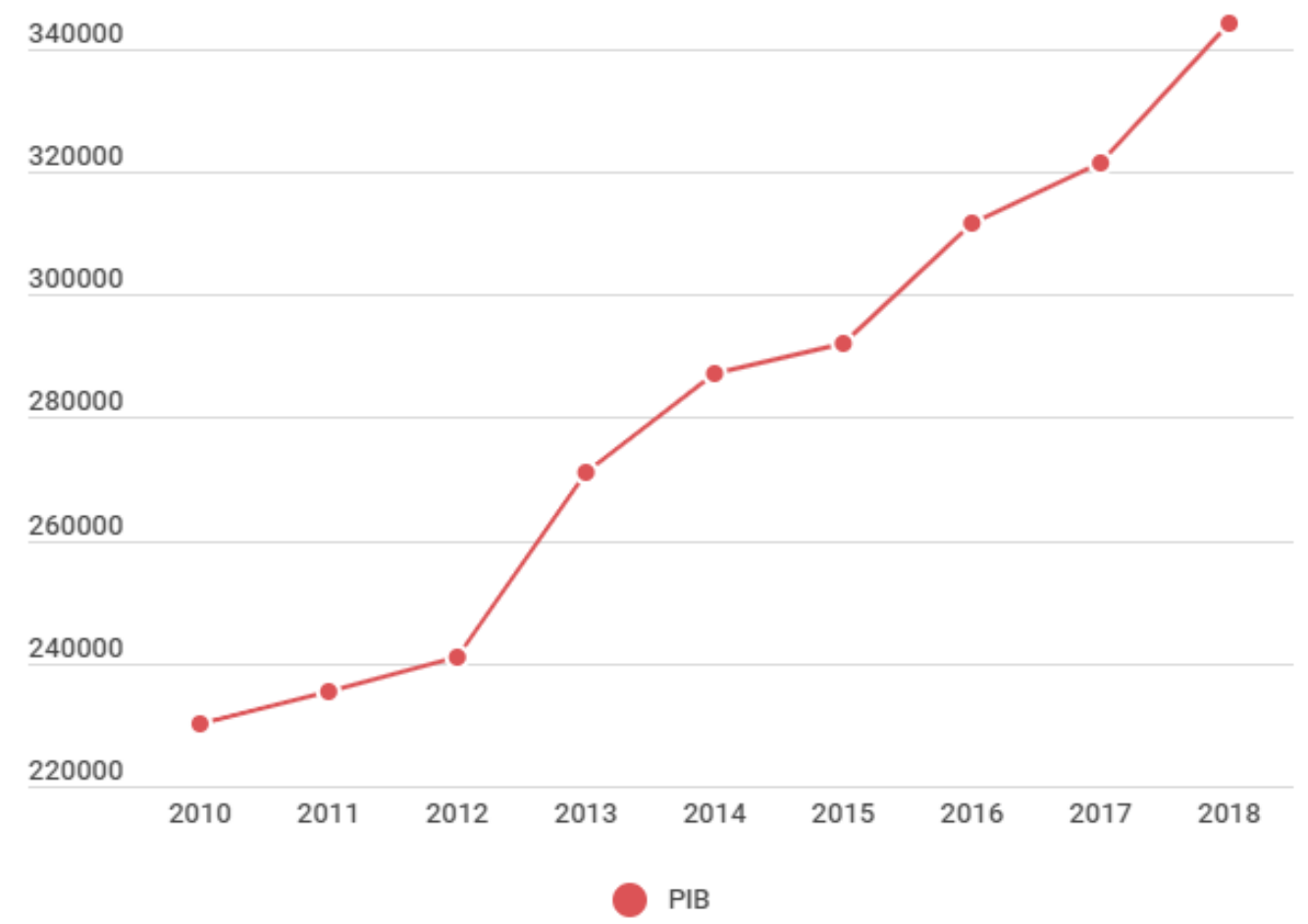

Fuente: SEMAGRO/MS (s.d.) - preparado por el autor.

Como se puede ver en el gráfico, la ciudad de Porto Murtinho ha tenido un aumento creciente en su PIB desde 2010, y la ruta bioceánica ha tenido influencia directa desde 2016. Aunque parece haber un gran aumento de la ciudad, es necesario compararlo con otras localidades del estado de Mato Grosso do Sul. 
Figura 7 - PIB per cápita y ranking en Mato Grosso do Sul(MS) de Porto Murtinho 2010 2018

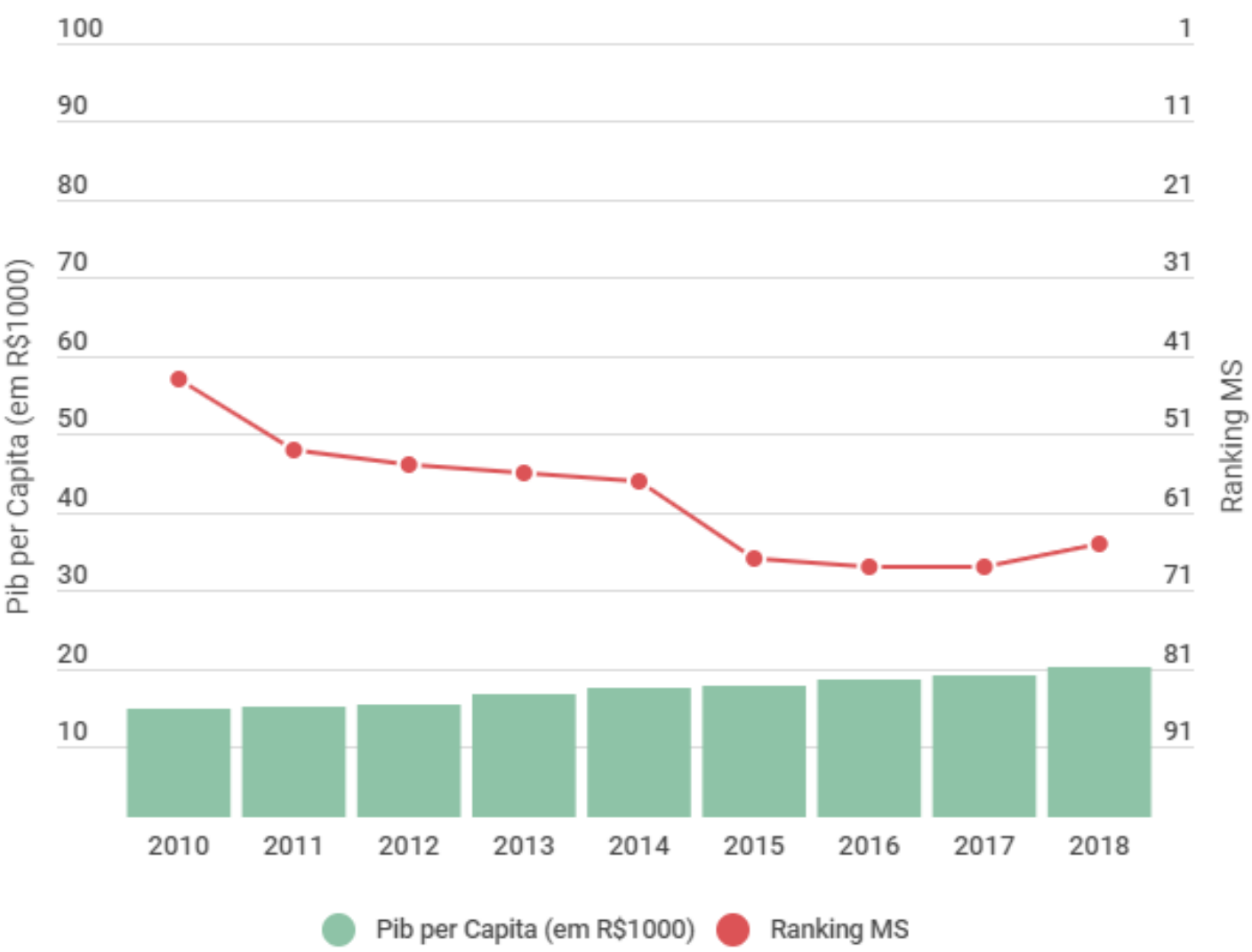

Fuente: SEMAGRO/MS (s.d.) - preparado por el autor.

El presente gráfico del PIB per cápita acompaña el aumento visto del PIB (Figura 6), no obstante, el ranking de la ciudad dentro del estado, aun con el aumento creciente del PIB, solo presenta caídas, significando que el desarrollo no fue de la ciudad de Porto Murtinho, sino del estado de Mato Grosso do Sul, que benefició la ciudad durante el período de 2010 a 2015.

A partir del año de 2016 la ruta entra en escena y provoca directamente el desarrollo en la ciudad de Porto Murtinho, es decir, el aumento visto en el PIB (cuadro 6) y en el PIB per cápita (cuadro 7) de los años de 2016 a 2018 tiene influencia directa de la construcción del corredor.

En los números, el PIB per cápita de Porto Murtinho aumentó alrededor de $\mathrm{R} \$ 3.000,00$ (de 14.979, 14 a 17.682, 51) en el período de 2010 a 2015, sin embargo, hubo una caída en el ránking de las ciudades de MS de 23 posiciones (de 44으 para 67으). A mediados de 2016, el corredor comienza a ser desarrollado y Porto Murtinho casi cierra el año con un aumento de casi $\mathrm{R} \$ 1.000,00$ en el PIB per cápita (de 17.682,51 para $18.669,80$ ) y de la caída de solo en el ranking (67으 para 68ㅇ). De ahora en adelante, la ciudad comienza a tener un crecimiento real.

En 2017, no hubo una caída en el ranking como había pasado en los últimos los siete años y todavía hubo un pequeño aumento en su PIB per cápita (de 18.669,80 para 19.038,73). Finalmente, en 2018, el aumento fue de más que $R \$ 1.000,00$ (de $19.038,73$ para $20.154,55$ ) y el comienza a subir en el ranking de las ciudades (de 68ㅇ para 65ㅇ). 
Con base a los datos antes mencionados, la ciudad de Porto Murtinho creció en 3 (tres) años lo que antes de que hubía tomado 7 (siete) años para alcanzar, quedàndo clara así, la gran influencia del corredor bioceánico para la ciudad.

Figura 8 - PIB Porto Murtinho por sector 2010 - 2018

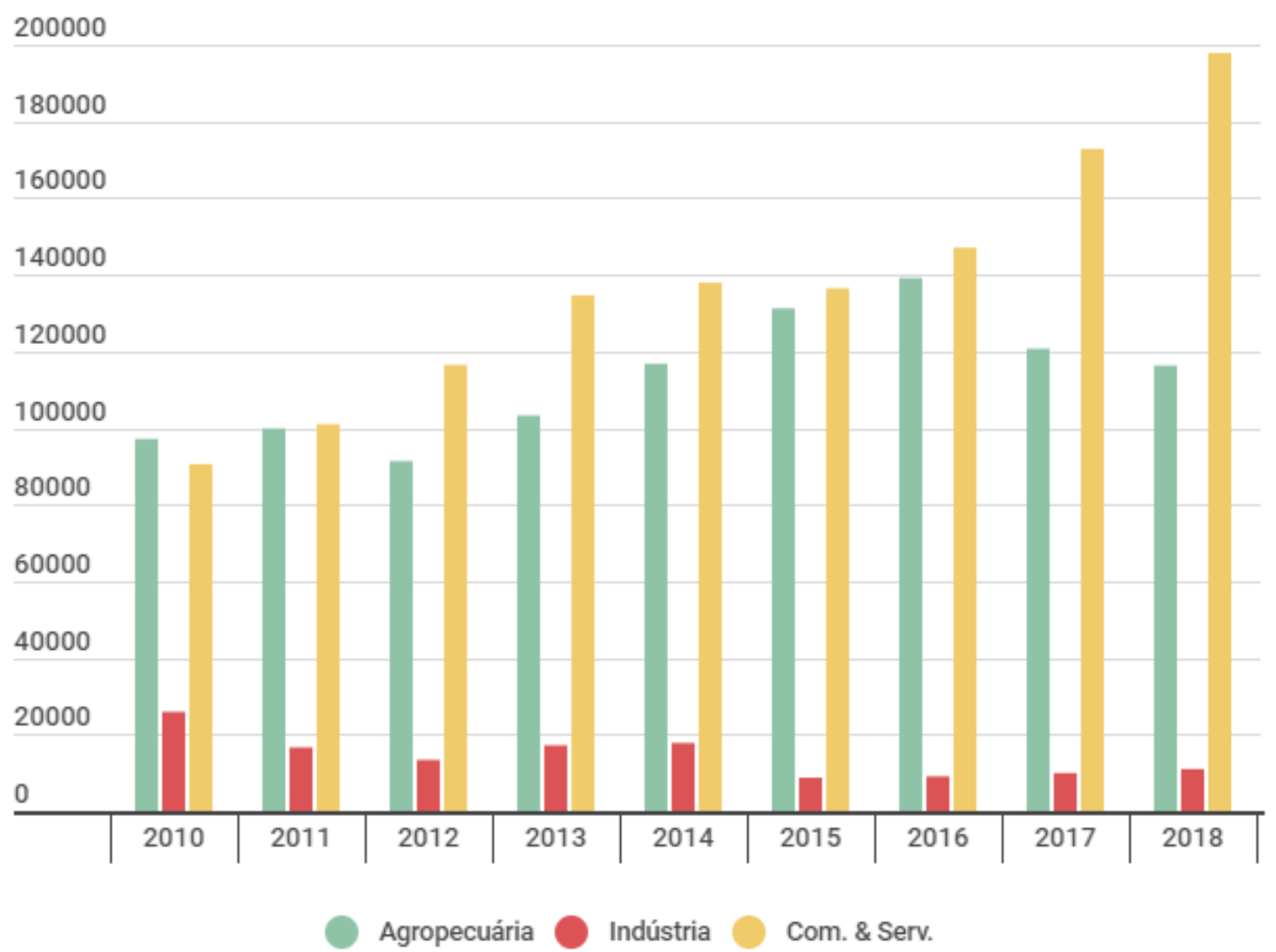

Fuente: SEMAGRO/MS (s.d.) - preparado por el autor.

A partir del gráfico, es posible visualizar qué tipos de desarrollos tuvo la ciudad de Porto Murtinho. Es importante señalar la baja relevancia del sector industrial para la ciudad, que, en el período 2010-2015, tuvo una caída aproximada de $\mathrm{R} \$$ 17.000,00 en números absolutos y, en el período 2010-2018, la relevancia del sector para el PIB pasó de 12,13\% a 3,37\%. A pesar de presentar crecimiento en números absolutos entre 2016-2018, su relevancia es cada vez menor para la ciudad.

La agricultura y la ganadería tienen números insaciables debido a su naturaleza, ya que el cosecha depende de factores externos para ser buena o mala. Sin embargo, se demostró que el PIB solo presentó crecimiento en el período analizado (figura 6), es decir, es importante analizar la relevancia de la agricultura para la ciudad.

En 2010, la agricultura y la ganadería representaban el 45,48\% del PIB de Porto Murtinho, y alcanzaron su punto máximo en 2015 con el 47,47\% y después solo mostraron descensos cerrando en 2018 con un 35,76\%. Al comparar su relevancia con los números absolutos presentes en el gráfico, se percibe que no solo se debió a la caída de los números del sector, sino también al gran aumento del sector de comercio y los servicios. 
El sector terciario muestra un alto crecimiento en los primeros seis años (2010-2015), sin embargo, con la llegada del corredor, su incremento en los siguientes tres años (2016-2018) es mayor que el primer espacio de tiempo y la relevancia del sector pasó del 42,39\% en 2010 a 60,86\% en 2018. Es decir, el crecimiento del comercio y los servicios en Porto Murtinho se duplicó por la influencia de la ruta.

\section{PERSPECTIVAS DE FUTURO Y ADVERSIDADES}

A partir de los datos presentados, los puntos positivos de la llegada del Corredor Bioceánico son evidentes. El alto crecimiento de la ciudad de Porto Murtinho, principalmente en el sector terciario, una salida para el mar para Paraguay, la reducción de costes en el transporte de marcancías, además de la previsibilidad en el trayecto, dándoles más seguridad para los que transitan por la ruta. Es nuclear recordar, además, de las ciudades cercanas a las que participan directamente en la ruta, que también se beneficiarán directa e indirectamente por el corredor.

A pesar de todo lo positivo, no se puede ignorar las dificultades y los problemas oriundos de ese gran desarrollo repentino. Para los gobiernos, como los de Brasil, que son exigentes con relación al plan director, se debe cuidar no dejar que las limitaciones del plan aparten a las inversiones necesarias en las ciudades. También se debe cuidar los tributos, especialmente los impuestos estatales y municipales, que pueden ser severos y terminar por alejar a las empresas del país.

Vale la pena recordar que, con la integración posibilitada por la ruta, la empresa que va a invertir en América Latina irá a escoger el país que más ventajas le dé para establecerse. No se trata de dar subsidios para las compañías o los empresarios, sino hacer posible un ambiente menos burocrático, pues, aunque hay altos impuestos, caso los costes con la burocracia y el tiempo dispendido con esta sean menores, es posible atraer a los inversores.

Importante destacar que el inversor no es solo la empresa con gran capital, sino que un pequeño empresario local que emplea una o dos personas también es considerado un inversor, puesto que, al fin y al cabo, genera empleos, renta y ofrece un servicio para la sociedad. Para este tipo de inversor, los costos burocráticos, la mayoría de las veces, son mayores que los costes tributarios, por tanto, cabe los gobiernos tener la debida atención a esta cuestión.

Debe cuidarse mucho con las leyes que tratan sobre la competencia, pues con la supresión de esta, podrán ser instalados monopolios y cárteles que obstaculizarán no solo las nuevas compañías e inversores, sino que también disminuirán el desarrollo del lugar.

En varias ocasiones, los cárteles y monopolios surgen bajo la premisa del proteccionismo. La idea de proteger la producción nacional para preservar o para generar trabajos dentro del país va total de satisfacer al objetivo de la ruta de integración. El proteccionismo nunca ha sido la solución para la generación de riqueza o desarrollo, por el contrario, es la razón del aumento de los precios de los productos y la pérdida de su calidad, ya que los productos externos son mucho más caros o incluso se les impide ingresar al país.

Otra forma de promoverse el proteccionismo es a partir de tasas de importación. La ruta tiene como objetivo volver más baratos los precios de las mercancías a ser exportadas e importadas, por tanto, incluso con la forma de la actual coyuntura económica global, es muy ventajoso a las personas, incluso a los gobiernos, la reducción o extinción de las tasas de importación, una vez que la población tendría acceso a más productos, tecnología, alimentos, accesorios, ropas etc., por un bajo coste. 
Otro punto a destacar es la mano de obra. Por tratarse de un pueblo pasando por un gran desarrollo repentino, la fuerza de trabajo actual en Porto Murtinho no posee alta calificación, por lo tanto, se hace necesario calificar a las persoonas, sea por parte del gobierno, lo que está de hecho haciéndose por Serviço Brasileiro de Apoio às Micro e Pequenas Empresas (Sebrae) y Superintendência de Ciência e Tecnologia, Produção e Agricultura Familiar (Semagro), o por la experiencia de trabajo.

En lo referente a la UniRila, con el acercamiento académico entre los países del corredor, será posible la creación de programas del intercambio entre las universidades, formación de grupos de investigación con las academias en conjunto y también crear una base para la producción del conocimiento futuro en medio a perspectivas variadas que se ponen a favor del interés común.

\subsection{La moneda corriente}

Con un tráfico más grande de gente entre los países, surge el problema del cambio de moneda. En Brasil, la circulación de cualquier moneda que no sea el real está prohibida. Como esta moneda es exclusiva de Brasil, queda pendiente una relajación en la legislación para permitir una mayor facilidad para los extranjeros que utilizan principalmente el dólar. Dicha prohibición se origina del Decreto-Ley n. 857 (BRASIL, 1969):

Art 1으o nulos de pleno direito os contratos, títulos e quaisquer documentos, bem como as obrigações que exequíveis no Brasil, estipulem pagamento em ouro, em moeda estrangeira, ou, por alguma forma, restrinjam ou recusem, nos seus efeitos, o curso legal do cruzeiro.

El artículo se ocupa de la moneda corriente en aquel entonces (cruzeiro), pero con la llegada del real, hubo el cambio solamente del curso de la moneda, sin embargo, la ley continúa válida, impidiendo así que las transacciones en el país sean hechas en monedas extranjeras. Así siendo, es un obstáculo a ser superado y sería una libertad dada a los brasileños y a quiénes vienen a Brasil, de poder elegir la moneda que mejor le sirve en vez de imponer un uso forzado.

La base para la imposición de la moneda es la Ley de Gresham que afirma que "El dinero malo expulsa el dinero bueno" y para prevenir fraudes, según Rothbard, hay un error en la interpretación de esta ley, de forma que solo sucede en caso de que haya una valoración artificial de la moneda.

La ley de Gresham es válida solamente cuando hay un control de precios impuesto por el gobierno sobre el dinero. Lo que la Ley de Gresham realmente dice es que "el dinero que está artificialmente sobrevalorado por el gobierno sacará de circulación el dinero que está artificialmente subvalorado". (ROTHBARD, 2013 p. 24).

En Argentina es posible intercambiar hasta US\$200 por persona en canales gubernamentales, en medio de la crisis y la población argentina utiliza esta medida como seguridad para no devaluar su dinero. De esta forma se demuestra que, sin el curso forzado de la moneda, las personas buscan la que les es más favorable, probando la interpretación de la Ley de Gresham hecha para Rothbard, es decir, lo que sucede es que el dinero bueno expulsa el dinero malo en condiciones de libre circulación.

Luego, el curso forzado de la moneda solo tiene la intención de control de parte del Estado sobre todas las transacciones financieras en el país. En caso de que haya ley posterior que permita la circulación y el uso de moneda extranjera dentro del territorio nacional, no sólo facilitaría la inversión extranjera, sino que la población también adoptaría la moneda que mejor le conviene. 
En suma, frente a la rotura de las fronteras, el avance de la tecnología y después de los objetivos de la ruta de la integración, es interesante un cambio en la legislación monetaria para permitir el curso de otras monedas en territorio nacional, dejando a criterio de las personas cuál moneda utilizar y, por otra parte, de los establecimientos comerciales qué monedas aceptar. A los efectos de la seguridad jurídica, es posible obligar a dichos sitios, por ley, a anunciar de antemano qué tipos de billetes son aceptados por ellos.

\section{CONSIDERACIONES FINALES}

Ante todo lo expuesto a lo largo del presente trabajo, se puede concluir que ell Corredor Bioceánico es una gran inversióon, que conlleva importantes impactos positivos acompañado de diversas adversidades a ser enfrentadaas por la población, los gobiernos y los empresarios locales e internacionales.

En a lo que se refiere a Brasil, será posible observar un desarrollo concentrado en Mato Grosso do Sul, especialmennte en Porto Murtinho, puesto que el país posee una economía bien diversificada debido a su gran territorio, sin embargo, es un hechho que la ruta facilitará y reducirá los costes para todas las regiones del país.

Para Paraguay, con su tamaño menor y carencia de salidas al mar, el corredor representa un puente para el desarrollo del país agraviado por su geografía por posibilitar no solo la mejora de sus relaciones con China, Brasil, Chile y Argentina, así como el inicio de las nuevas rutas comerciales con diversos otros países.

Junto a una buena administración pública,-se entiende por "buena administración" aquella que promueve la simplicidad para los empresarios, es decir, sin endurecer la actividad económica por medio de burocracia, sin proveer los subsidios para la producción, que podrían causar la creación de imperfecciones de mercado.

\section{REFERENCIAS}

BRASIL. Decreto-Lei n. 857, de 11 de setembro de 1969. Consolida e altera a legislação sôbre moeda de pagamento de obrigações exequíveis no Brasil. Diário Oficial da União, Brasília, DF, 11 sept. 1969. Disponible en: https://www.planalto.gov.br/ccivil_03/decreto-lei/Del0857.htm. Acceso el: 14 abr. 2021.

CAMPOS, Luana; FARIA, Alcides. Rota Bioceânica - o que é e seus impactos diretos e indiretos. ECOA, Campo Grande, 2020. Disponible en: https://ecoa.org.br/rota-bioceanica-o-que-e-e-seus-impactosdiretos-e-indiretos/. Acceso el: 12 ene. 2021.

HUERTA DE SOTO, Jesús. A escola austríaca. 2. ed. Tradução de André Azevedo Alves. São Paulo: Instituto Ludwig von Mises Brasil, 2010.

IORIO, Ubiratan. Ação, tempo e conhecimento: a escola austríaca. 2. ed. São Paulo: LVM Editora, 2011.

MISES, Ludwig Von. Ação humana: um tratado de Economia. Campinas: Vide, 2015.

ROTHBARD, Murray N. O que o governo faz com o nosso dinheiro. Traducción de Leandro Augusto Gomes Roque. 3. ed. São Paulo: Instituto Ludwig Von Mises Brasil, 2013.

SECRETARIA DE ESTADO DE MEIO AMBIENTE, DESENVOLVIMENTO ECONÔMICO, PRODUÇÃO E AGRICULTURA FAMILIAR [SEMAGRO]. Contas regionais relatórios do PIB. SEMAGRO, Campo Grande, 
[s.d.]. Disponible en: http://www.semagro.ms.gov.br/contas-regionais-relatorios-do-pib. Acceso el: 12 ago. 2021.

THE OBSERVATORY OF ECONOMIC COMPLEXITY [OEC]. OEC, 2019. The best way to explore trade data. Disponible en: https://oec.world. Acceso el: 12 ago. 2021.

\section{Sobre los autores y la autora:}

Felipe Figueiredo Fernandes Brites: Estudiante de Derecho en la Universidad Católica Dom Bosco (UCDB). Investigador del Programa Institucional de Becas de Iniciación Científica (PIBIC) de la UCDB; y en el área de Derecho y Desarrollo Local, en el área de Derecho Internacional y Derecho Económico. Miembro del grupo de investigación sobre Patrimonio Cultural, Derecho y Diversidad, desde 2017. E-mail: felipe.fig.fer.brites@gmail.com, Orcid: https://orcid.org/0000-0001-8722-6755

Michel Constantino: Estudiante de postdoctorado en Políticas Públicas en la Fundación Getulio Vargas (FGV). Doctor en Economía por la Universidad Católica de Brasilia (UCB). Máster en Desarrollo Local por la Universidad Católica Dom Bosco (UCDB). Grado en Administración de Empresas por la Universidad Anhanguera-UNIDERP. Profesor en los programas de doctorado en Ciencias Ambientales y Sostenibilidad Agropecuaria y en Desarrollo Local. Investigador en el área de Políticas Públicas, Economía y Econometría (Métodos Cuantitativos). Investigador invitado en el Instituto de Investigación Económica y Aplicada (IPEA). Academic Board Member: Journal of Cleaner Production (Elsevier). Editor asociado de la Economic Analysis of Law Review. Líder del Grupo de Investigación del Consejo Nacional de Desarrollo Científico y Tecnológico (CNPq) Science With R. Coordinador del PEIEX - Programa de Exportación do Gobierno Federal (APEX). Coordinador del Doctorado y Máster en Desarrollo Local de la Universidad Católica Dom Bosco. E-mail: michel@ucdb.br, Orcid: http://orcid.org/0000-0003-2570-0209

Arlinda Cantero Dorsa: Realizando prácticas postdoctorales en Desarrollo Local en el Centro Universitario Augusto Motta (UNISUAM). Doctora en Lengua Portuguesa por la Pontificia Universidad Católica de São Paulo (PUC-SP). Máster en Comunicación y Letras por la Universidad Presbiteriana Mackenzie (UPM). Grado en Letras/Francés por la Facultad Dom Aquino de Filosofía, Ciencias y Letras; y en Pedagogía por la Facultad de Educación, Ciencias y Letras de Urubupungá (FECLU). Profesora titular de la Universidad Católica Dom Bosco (UCDB), en el curso de Derecho. Profesora y vicecoordinadora del Máster y Doctorado en Desarrollo Local de la UCDB. Miembro del Instituto Histórico e Geográfico de Mato Grosso do Sul (IHG-MS). Líder del Grupo de Investigación en Patrimonio Cultural, Derecho y Diversidades y subdirectora del Grupo de Investigación y Estudios en Tecnología Educativa y Educación a Distancia (GETED) de la UCDB. Coordina el proyecto Ruta Bioceánica y UniRila (Red Universitaria de la Ruta de Integración Latinoamericana): Conociendo el Potencial Existente como Nuevos Espacios de Desarrollo y Prácticas Innovadoras - Continuidad; y el Proyecto Gobernanzas Migratorias en Brasil: Desafíos Contemporáneos en la Agenda 2030 - Organización de las Naciones Unidas (ONU). E-mail: acdorsa@ucdb.br, Orcid: http://orcid.org/0000-0002-1120-0273 